\title{
Asymptomatic superficial siderosis after posterior fossa tumor resection: illustrative case
}

\author{
Anshit Goyal, MBBS, MS, Cody L. Nesvick, MD, Joshua A. Spear, MD, and David J. Daniels, MD, PhD \\ Department of Neurologic Surgery, Mayo Clinic, Rochester, Minnesota
}

BACKGROUND Superficial siderosis of the central nervous system is a rare syndrome notable for the presence of hemosiderin deposition due to chronic, repetitive hemorrhages into the subarachnoid space.

OBSERVATIONS The authors presented a case of superficial siderosis in a 14-year-old girl. It arose as a late postoperative complication after resection of a medulloblastoma. Despite the patient being asymptomatic, surveillance imaging demonstrated diffuse hemosiderin deposition within the cerebellar folia and cisternal segments of cranial nerves VII and VIII on gradient echo (GRE) sequences. Formal audiometric testing demonstrated bilateral loss of high-frequency tone recognition consistent with early sensorineural hearing loss. A pseudomeningocele due to multiple dural defects was identified as the likely cause, and definitive surgical repair was performed. Intraoperatively, the presence of blood-tinged cerebrospinal fluid confirmed a diagnosis of superficial siderosis.

LESSONS This case highlighted the potential need to routinely include GRE or susceptibility-weighted sequences in postoperative surveillance imaging after resection of pediatric posterior fossa tumors.

https://thejns.org/doi/abs/10.3171/CASE2174

KEYWORDS superficial siderosis; posterior fossa; pediatric; dural closure; durotomy; pseudomeningocele; cerebellar tumor; medulloblastoma; hearing loss

Superficial siderosis of the central nervous system (CNS) is a rare disorder characterized by subpial hemosiderin deposition due to chronic slow or repeated acute hemorrhages into the subarachnoid space. It can lead to sensorineural hearing loss, cerebellar ataxia, and pyramidal deficits and may be associated with multiple cranial neuropathies. Previously reported etiologies include dural defects, tumor-related hemorrhage, vascular abnormalities, and intradural surgery. ${ }^{1-4}$ With the improvement in survival in pediatric cerebellar tumors, the disorder may occasionally arise as a late postoperative complication after resection. ${ }^{3}$ The diagnosis is generally confirmed on susceptibility-weighted magnetic resonance imaging (MRI), which demonstrates hemosiderin deposition along the brainstem, cerebellum, and spinal cord. ${ }^{5,6}$ We describe a case of late, asymptomatic superficial siderosis that was first detected on surveillance imaging after resection of a medulloblastoma.

\section{Illustrative Case}

The case involved a 14-year-old girl with a history of medulloblastoma. Imaging demonstrated a partially contrast-enhancing fourth ven- tricular lesion and early ventriculomegaly (Fig. 1). She underwent external ventricular drain placement and tumor resection via suboccipital craniotomy and telovelar approach. The dura was close primarily with a bovine xenograft and application of dural sealant. Postoperatively, she was neurologically intact. Postoperative imaging demonstrated a small amount of residual tumor. Histopathology confirmed a non-wingless-activated, non-sonic hedgehog medulloblastoma. A second-look surgery was performed to obtain gross-total resection, and the same dural graft was reapproximated with silk sutures. Immediate postoperative MRI demonstrated no evidence of residual tumor but was notable for a small, posterior soft tissue fluid collection, which represented a small pseudomeningocele. Our patient subsequently underwent standard-risk chemotherapy and craniospinal radiation with posterior fossa boost (craniospinal irradiation: 18 Gy in 10 fractions; posterior fossa boost: 36 Gy in 17 fractions).

Interval imaging produced negative results for recurrent tumor but demonstrated an increase in the size of the posterior soft tissue fluid collection and a dural defect over the site of surgical repair, again consistent with a pseudomeningocele (Fig. 2A). Gradient

ABBREVIATIONS CNS = central nervous system; CSF = cerebrospinal fluid; GRE = gradient echo; MRI = magnetic resonance imaging. INCLUDE WHEN CITING Published May 3, 2021; DOI: 10.3171/CASE2174.

SUBMITTED February 2, 2021. ACCEPTED February 10, 2021.

(c) 2021 The authors, CC BY-NC-ND 4.0 (http://creativecommons.org/licenses/by-nc-nd/4.0/). 

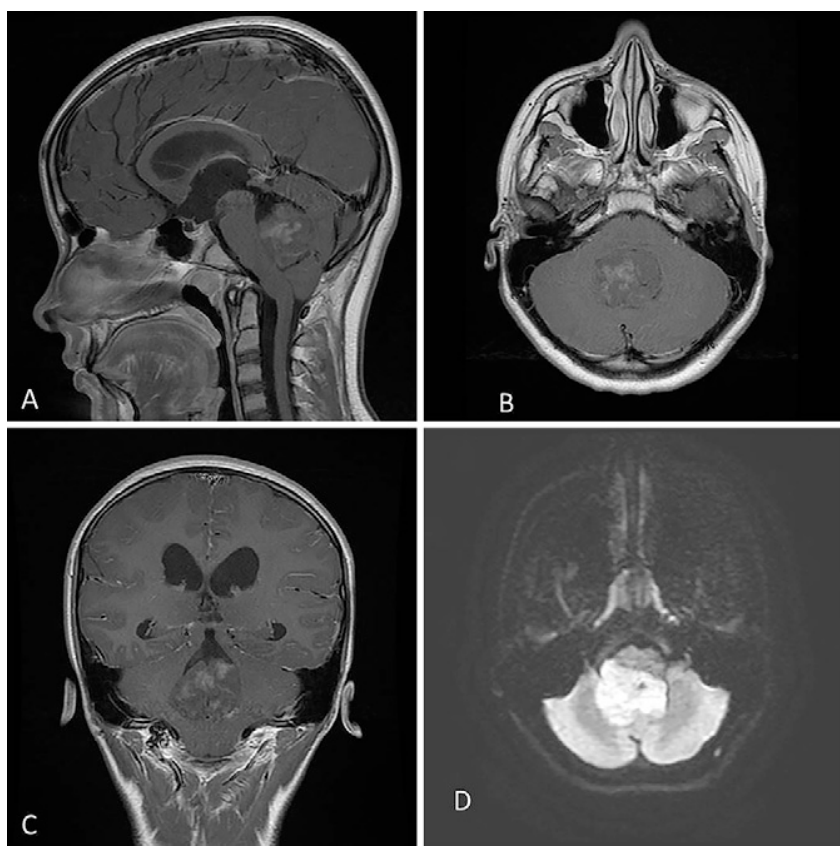

FIG. 1. Preoperative MRI demonstrating $(\mathbf{A}-\mathbf{C})$ a heterogeneous, contrast-enhancing mass in the fourth ventricle with (D) restricted diffusion, consistent with a medulloblastoma.

echo (GRE) T2-weighted imaging performed as part of the scans demonstrated loss of susceptibility along the cerebellar vermis that remained stable on serial imaging (Fig. 2B). After initiation of maintenance chemotherapy, audiometry performed to monitor ototoxicity demonstrated World Health Organization grade 1 hearing loss that remained stable on serial follow-up examinations. Nearly 7 months after cessation of maintenance chemotherapy, surveillance imaging demonstrated hemosiderin deposition within the posterior fossa along the cerebellar folia and brainstem, including cranial nerves VII and VIII (Fig. 3). Although there was no subjective hearing loss, a repeat audiogram was obtained given the imaging findings, which demonstrated World Health Organization grade 2 sensorineural hearing loss.
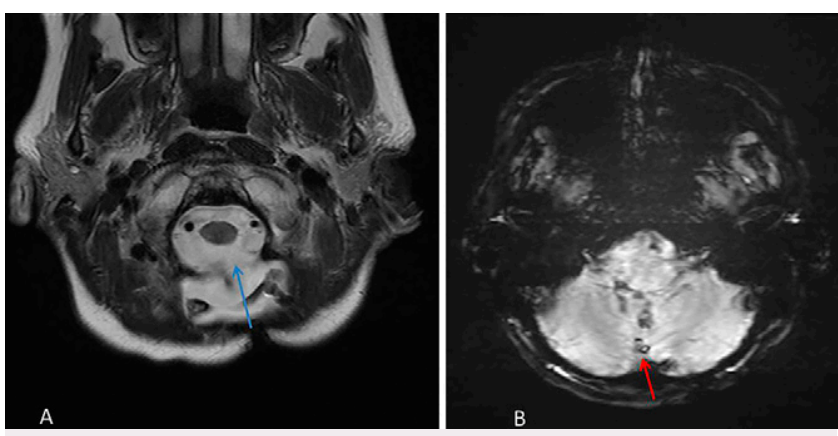

FIG. 2. A: Postoperative follow-up imaging demonstrated an epidural soft tissue fluid collection with an overlying dural defect (blue arrow). B: Serial imaging demonstrated stable foci of GRE findings (red arrow) consistent with hemosiderin deposition along the cerebellar vermis.
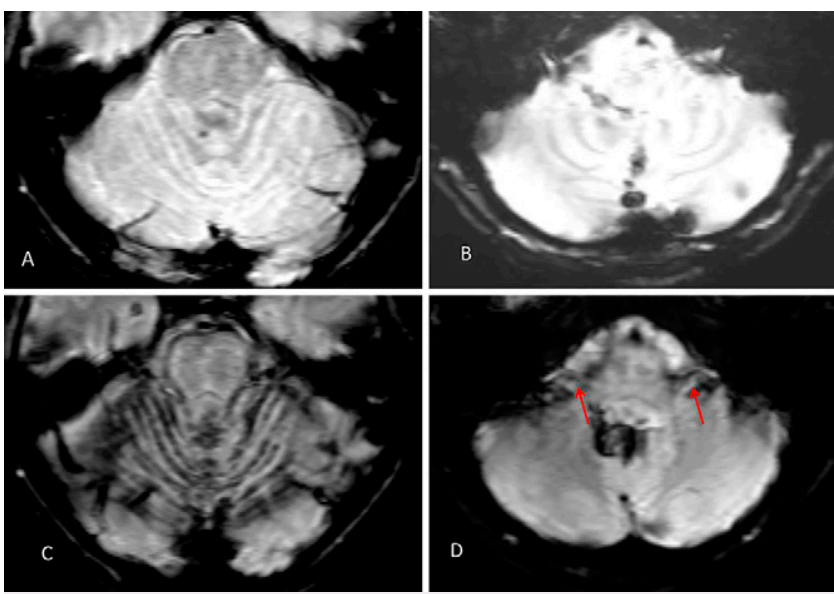

FIG. 3. Serial GRE T2-weighted imaging 15 months (A and B) and 18 months ( $C$ and $\mathbf{D}$ ) after surgery demonstrated increased hemosiderin deposition along the cerebellar folia ( $\mathbf{C}$ vs $\mathbf{A}$ ) and cranial nerves VII and VIII (red arrows, D vs B).

Because of the constellation of imaging findings accompanied by objective evidence of hearing decline, a diagnosis of superficial siderosis was made. Given the potential contribution of the pseudomeningocele to these findings, a pseudomeningocele repair was performed. Intraoperatively, blood-tinged cerebrospinal fluid (CSF) was immediately encountered upon fascial opening. Upon removal of the bone flap, a large clot was present over the duraplasty, and following removal of the clot, multiple defects within the dural patch were readily apparent (Fig. 4). After copious irrigation to clear residual clot, a local pericranial graft was harvested and a watertight duraplasty was performed with dural sealant augmentation. CSF cytology results were negative for neoplastic cells.

\section{Discussion}

Chronic hemorrhage into the subarachnoid space is the most common cause of superficial siderosis, although a source is not always identifiable. ${ }^{7}$ Previously demonstrated etiologies include chronic tumor-related hemorrhage, vascular malformations, dural defects, nerve root avulsions, and even brachial plexus injuries. ${ }^{3,8-14}$ The pathophysiology is said to be linked to overloading of the ferritin biosynthesis pathway, which serves as a neuroprotective mechanism by preventing the conversion of heme to hemosiderin. ${ }^{15}$ Chronic, repetitive hemorrhage may exhaust this reserve and lead to hemosiderin formation and oxidative damage to tissues. ${ }^{16}$ Acute subarachnoid hemorrhage only transiently activates heme oxygenase-1, a key enzyme required to convert heme into hemosiderin, which possibly explains the lack of superficial siderosis in these patients. ${ }^{17}$

Several reports in the literature have demonstrated the link between both low- and high-flow dural defects and superficial siderosis, including three previously published cases from our institution, which were also linked to dural defects after posterior fossa surgery. ${ }^{18}$ In a review of superficial siderosis cases published in the literature, Fearnley et al. identified a dural etiology in $47 \%$ (34/63) of cases. ${ }^{1}$ In this case, the dural defects were presumably due to slow erosion of the synthetic graft used for duraplasty. In instances in which superficial siderosis is clearly related to a dural defect, definitive dural closure can halt disease progression and prevent further 


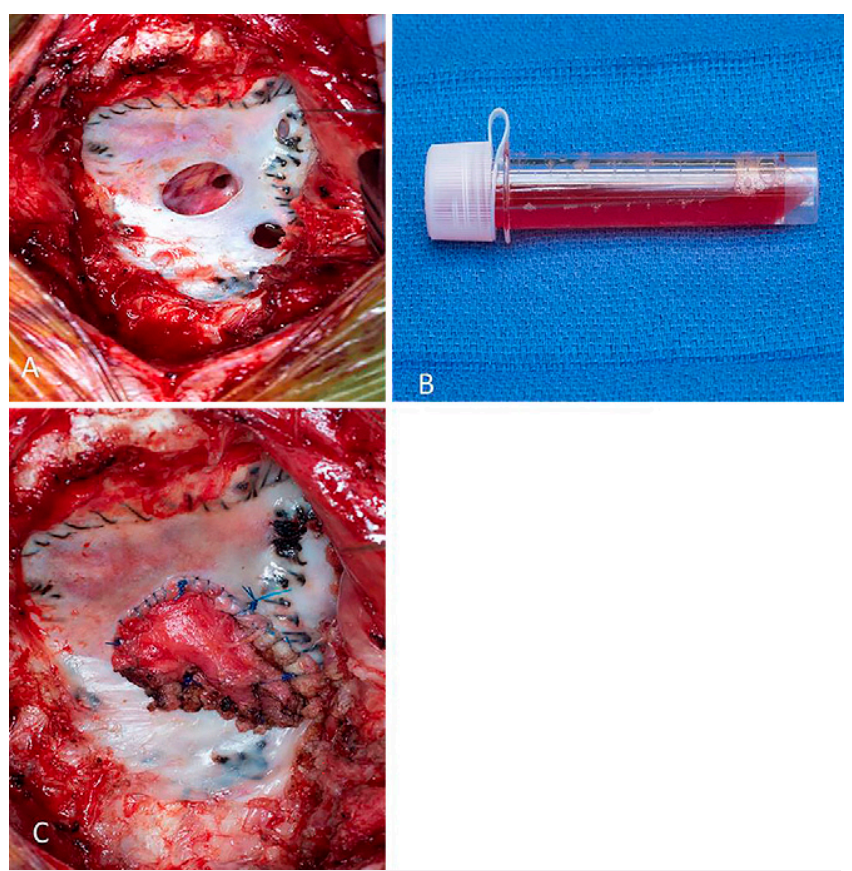

FIG. 4. Intraoperative photos. A: Multiple dural defects within the synthetic dural graft. B: Blood-tinged CSF collected for laboratory evaluation. C: Watertight duraplasty performed using a pericranial graft.

neurological dysfunction. ${ }^{18}$ The mechanism of superficial siderosis due to dural defects has been hypothesized to be either CSF hypotension resulting in red blood cell extravasation from engorged blood vessels, or friable blood vessels leading to chronic, repetitive microhemorrhages. ${ }^{18}$

Superficial siderosis has been previously reported after surgery in the posterior fossa, especially after resection of cerebellar tumors. ${ }^{3,13,19,20}$ In addition to the negative pressure on blood vessels caused by CSF leaks and pseudomeningocele formation, it is possible that having a surgical wound exposed to the subarachnoid space through a dural defect may contribute to chronic hemosiderin deposition in the brain. ${ }^{3,18}$ Together, these points highlight the importance of achieving as watertight a dural closure as possible at surgery to minimize the risk of not only wound complications but also superficial siderosis. It has been shown that graft duraplasty is usually associated with lower rate of CSF leaks as compared to primary dural closure; however, this association is somewhat disputed. ${ }^{21}$ Biological graft material may be superior to synthetic substitutes because of better dural integration by promoting fibroblast ingrowth and neovascularization. ${ }^{22}$ Furthermore, use of autologous grafts (pericranium, fascia lata) is associated with a lack of immunogenicity and lower rates of CSF leakage and wound complications, as compared to other biological or synthetic graft material. ${ }^{21-25}$ However, use of autologous grafts may be limited by donor site morbidity. ${ }^{23}$

\section{Observations}

The patient presented in this study was asymptomatic, although surveillance MRI suggested the diagnosis of superficial siderosis.
This condition usually presents in a delayed fashion, often many years after initial surgery. ${ }^{20}$ Early intervention may prevent disease progression; therefore, this study highlights the necessity of susceptibility-weighted imaging or GRE sequences when performing surveillance imaging for patients treated for posterior fossa tumors. Conventional spin-echo and fast spin-echo T2weighted images may also demonstrate hypointensity that is consistent with hemosiderin deposition, but they are less sensitive compared to susceptibility-weighted imaging and GRE. ${ }^{26}$ The cerebellum, brainstem, and cisternal cranial nerve segments are the most commonly affected structures, ${ }^{27}$ although iron deposition may also be observed more diffusely within the CNS. The cerebellar folia and cranial nerve VIII are especially vulnerable to hemosiderin deposition because of the presence of Bergmann glia, which are generally responsible for accelerated ferritin synthesis. $^{18,27}$

\section{Lessons}

Superficial siderosis may be a late, asymptomatic, postoperative complication after resection of tumors in the posterior fossa. Susceptibility-weighted imaging should be routinely performed in surveillance imaging of patients with this condition because early detection is important to allow timely intervention, even in asymptomatic cases, when an underlying surgically correctable cause can be identified. This approach is key to halting disease progression and further neurological dysfunction.

\section{References}

1. Fearnley JM, Stevens JM, Rudge P. Superficial siderosis of the central nervous system. Brain. 1995;118(pt 4):1051-1066.

2. Kole MK, Steven D, Kirk A, Lownie SP. Superficial siderosis of the central nervous system from a bleeding pseudomeningocele. Case illustration. J Neurosurg. 2004;100(4):718.

3. Anderson NE, Sheffield S, Hope JK. Superficial siderosis of the central nervous system: a late complication of cerebellar tumors. Neurology. 1999;52(1):163-169.

4. Kumar N, Cohen-Gadol AA, Wright RA, et al. Superficial siderosis. Neurology. 2006;66(8):1144-1152.

5. Kumar N. Neuroimaging in superficial siderosis: an in-depth look. AJNR Am J Neuroradiol. 2010;31(1):5-14.

6. Uchino A, Aibe $\mathrm{H}$, Itoh $\mathrm{H}$, et al. Superficial siderosis of the central nervous system. Its MRI manifestations. Clin Imaging. 1997;21(4): 241-245.

7. Offenbacher $H$, Fazekas F, Schmidt R, et al. Superficial siderosis of the central nervous system: MRI findings and clinical significance. Neuroradiology. 1996;38(suppl 1):S51-S56.

8. Aquilina K, Kumar R, Lu J, Rawluk D. Superficial siderosis of the central nervous system following cervical nerve root avulsion: the importance of early diagnosis and surgery. Acta Neurochir (Wien). 2005;147(3):291-297.

9. Cohen-Gadol AA, Krauss WE, Spinner RJ. Delayed central nervous system superficial siderosis following brachial plexus avulsion injury. Report of three cases. Neurosurg Focus. 2004;16(5):E10.

10. Hsu WC, Loevner LA, Forman MS, Thaler ER. Superficial siderosis of the CNS associated with multiple cavernous malformations. AJNR Am J Neuroradiol. 1999;20(7):1245-1248.

11. Konya D, Peker S, Ozgen S, et al. Superficial siderosis due to papillary glioneuronal tumor. J Clin Neurosci. 2006;13(9):950-952.

12. Matsumoto $S$, Kang $Y$, Sato $S$, et al. Spinal meningeal melanocytoma presenting with superficial siderosis of the central nervous system. Case report and review of the literature. $J$ Neurosurg. 1998;88(5):890-894. 
13. Kumar A, Aggarwal S, Willinsky R, TerBrugge KG. Posterior fossa surgery: an unusual cause of superficial siderosis. Neurosurgery. 1993;32(3):455-457.

14. Kumar N, McKeon A, Rabinstein AA, et al. Superficial siderosis and CSF hypovolemia: the defect (dural) in the link. Neurology. 2007; 69(9):925-926.

15. Koeppen AH, Dickson AC, Chu RC, Thach RE. The pathogenesis of superficial siderosis of the central nervous system. Ann Neurol. 1993;34(5):646-653.

16. Koeppen AH, Michael SC, Li D, et al. The pathology of superficial siderosis of the central nervous system. Acta Neuropathol. 2008; 116(4):371-382.

17. Koeppen AH, Dickson AC. Tin-protoporphyrin prevents experimental superficial siderosis in rabbits. $J$ Neuropathol Exp Neurol. 2002;61(8):689-701.

18. Kumar R, Jacob JT, Welker KM, et al. Superficial siderosis of the central nervous system associated with incomplete dural closure following posterior fossa surgery: report of 3 cases. J Neurosurg. 2015;123(5):1326-1330.

19. Moreira NC, Nylander R, Briaukaite I, et al. Superficial siderosis: a case report. Medicina (Kaunas). 2011;47(6):320-322.

20. McCarron MO, Flynn PA, Owens $C$, et al. Superficial siderosis of the central nervous system many years after neurosurgical procedures. J Neurol Neurosurg Psychiatry. 2003;74(9):1326-1328.

21. Hale AT, Gannon SR, Zhao S, et al. Graft dural closure is associated with a reduction in CSF leak and hydrocephalus in pediatric patients undergoing posterior fossa brain tumor resection. J Neurosurg Pediatr. Published online November 29, 2019. doi: 10.3171/2019.9.PEDS1939

22. Azzam D, Romiyo P, Nguyen $T$, et al. Dural repair in cranial surgery is associated with moderate rates of complications with both autologous and nonautologous dural substitutes. World Neurosurg. 2018;113:244-248.

23. Abla AA, Link T, Fusco D, et al. Comparison of dural grafts in Chiari decompression surgery: review of the literature. J Craniovertebr Junction Spine. 2010;1(1):29-37.
24. Zhao Y, Chen L, Zhang J, et al. Duraplasty with cervical fascia autograft to reduce postoperative complications of posterior fossa tumor surgery with suboccipital midline approach. World Neurosurg. 2020;134:e1115-e1120.

25. Malliti M, Page P, Gury C, et al. Comparison of deep wound infection rates using a synthetic dural substitute (Neuro-Patch) or pericranium graft for dural closure: a clinical review of 1 year. Neurosurgery. 2004;54(3):599-604.

26. Wang J, Gong X. Superficial siderosis of the central nervous system: MR findings with susceptibility-weighted imaging. Clin Imaging. 2011;35(3):217-221.

27. Johans SJ, Swong KN, Burkett DJ, et al. Clival meningocele causing bilateral hearing loss in a child due to superficial siderosis of the central nervous system: case report. J Neurosurg Pediatr. 2018;21(5):498-503.

\section{Disclosures}

The authors report no conflict of interest concerning the materials or methods used in this study or the findings specified in this paper.

\section{Author Contributions}

Conception and design: Daniels, Goyal, Nesvick. Acquisition of data: Daniels, Goyal, Spear. Analysis and interpretation of data: Daniels, Nesvick. Drafting the article: Daniels, Goyal. Critically revising the article: Daniels, Goyal, Nesvick. Reviewed submitted version of manuscript: all authors. Approved the final version of the manuscript on behalf of all authors: Daniels. Administrative/technical/material support: Daniels. Study supervision: Daniels.

\section{Correspondence}

David J. Daniels: Mayo Clinic, Rochester, MN. daniels.david@mayo. edu. 